\title{
Saturation IHS Image Fusion: A New Method of Image Fusion
}

\author{
Sreelekshmi A. N. \\ Department of Computer Science \\ Sree Ayyappa College, Eramallikkara, \\ Chengannur, India
}

\begin{abstract}
Image fusion can be used as a tool to increase the spatial resolution. In that case the high resolution panchromatic imagery is fused with low-resolution often multi-spectral image data. The multispectral images are images created from the several narrow spectral bands. It contains all spectral (color information) details but not spatial details. Panchromatic images are single band images generally displayed as shades of gray. It contains all high spatial details (geometric) but not spectral details. Various fusion algorithms have been developed over the years. Image fusion methods can be broadly classified into two categories - spatial domain and transform domain methods. All these methods improve spatial or spectral resolutions. Hence, there is a requirement for development of newer techniques to fuse high resolution Cartosatseries data with high resolution of spatial and spectral details of all image data type
\end{abstract}

\section{Keywords}

Image fusion, pan chromatic image, multispectral image, spatial resolution, spectral resolution

\section{INTRODUCTION}

Indian Remote Sensing Satellites provides observations of the earth features at various spatial and spectral resolutions. With the availability of Cartosat series satellites data is available at $2.5 \mathrm{~m}$ and $1 \mathrm{~m}$ spatial resolutions.

It is desirable for many Remote sensing applications to have data in different spectral bands at higher spatial resolutions to provide improved classification accuracy. There are many approaches for improving the spatial resolutions like reducing the height of the orbit, reducing the pixel size of the CCD detector, etc... As all of these methods will have theoretical maximum limits, there is a requirement for improving the spatial resolution using ground processing methods. PAN sharpening/Image fusion is such technique to improve the spatial and spectral resolutions.

Image fusion is the process that combines information from multiple images of the same scene. The result of image fusion is a new image that retains the most desirable information and characteristics of each input image. Applied for Remote sensing images Image fusion / Resolution merge is the process of combining high resolution panchromatic image with the low resolution multispectral image to get high resolution multispectral imagery. Image fusion enables us to integrate imagery of different spatial resolutions [5].

\section{OVERVIEW OF THE PROPOSED METHOD}

The proposed method is the new panchromatic image is fused with multispectral image. The new panchromatic image is created by using apply the multiple linear regression. Then we get the synthetic panchromatic image. The advantage of the new panchromatic image is more spatially detailed one. Here we are applying the saturated compensated HIS image fusion method for applying image fusion. So fused with new panchromatic image and multispectral image finally we get the highly spatially and high spectrally detailed image as the output.

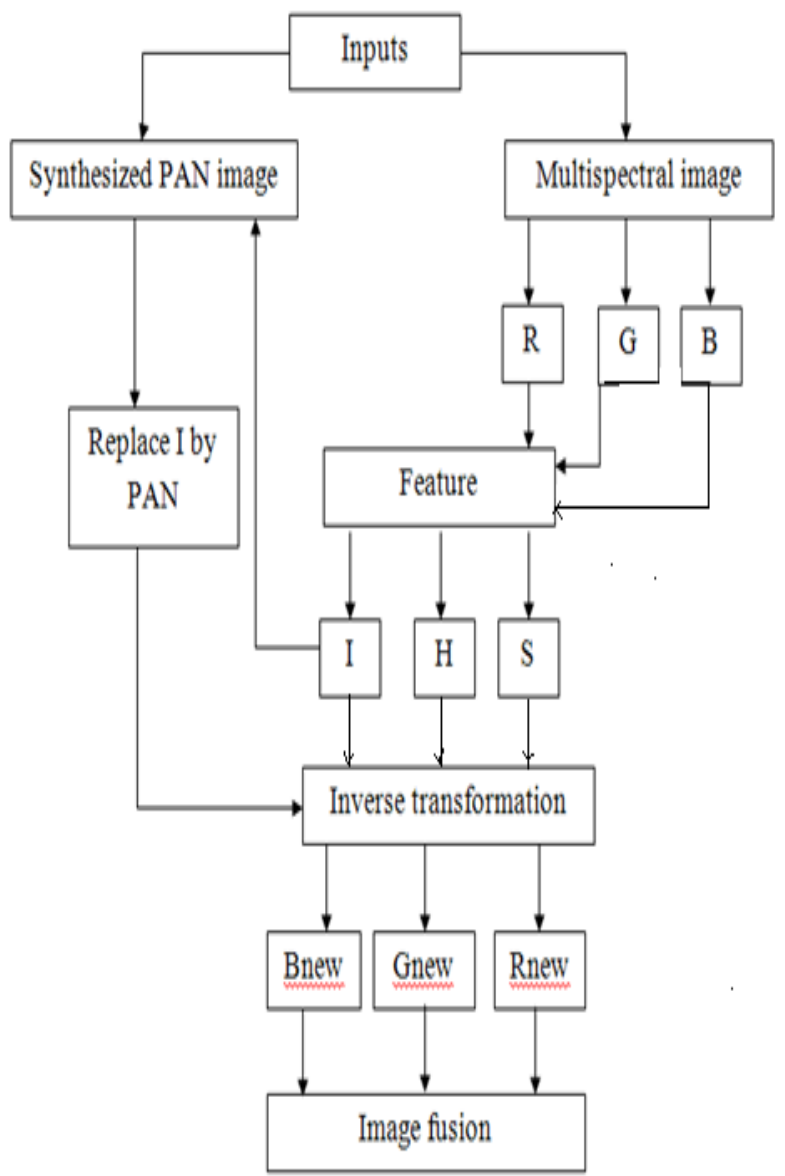

The proposed method is related to the multiple linear regression based one. The multispectral image is fused with panchromatic image then we get one image with especially high resolution and also spectrally high resolution image. The main differences of the other HIS methods with this are here not take directly as the panchromatic image.

The multiple linear regression equation is:

$$
\mathrm{Y}=\mathrm{B} * \mathrm{X}
$$

Where, 
' $\mathrm{B}$ ' is the matrix of $\mathrm{B} 1, \mathrm{~B} 2$ and $\mathrm{B} 3$. This is considered as the coefficient matrix of regression.

' $\mathrm{Y}$ ' is the panchromatic image matrix and ' $\mathrm{X}$ ' is the multispectral image matrix.

\subsection{To find the synthesized panchromatic image}

PAN image is $1 \mathrm{xN}$ matrix and that is equated to the three $3 \mathrm{xN}$ matrix of multispectral image and regression coefficients like $a, b$ and $c$ ( $1 \times 3$ matrix). Here we get the values of $a, b$ and $c$. This coefficient matrix is multiplied with multispectral image matrix finally we got one new image matrix, called synthesized PAN image.

In this method calculate to find the coefficient matrix of regression from this equation:

$\mathrm{B}=($ inverse $(\operatorname{transpose}(\mathrm{X})) *(\mathrm{X})) *(\operatorname{transpose}(\mathrm{X})) *(\mathrm{Y})$ Eqn (7)

From here we get one matrix that is multiplied with multispectral image finally we get one binary image, called synthesized panchromatic image. In the proposed method we use this synthesized panchromatic image is fused with multispectral image. Here the saturation compensated image fusion is applied for the image fusion.

The proposed method we will calculate the regression coefficients and that value is equating to the original PAN image. Then we get the coefficients, that value is multiplied by the multispectral image finally we will get one new image called synthesized PAN image. This new PAN image is used to image fusion.

\section{PROPOSED METHOD}

According to Chen et al., 2003 in the IHS transformation image fusion, the Intensity (I), the spatial component and the Hue $(\mathrm{H})$ and the Saturation (S), the spectral components of an image are generated from the RGB image. The Intensity (I) component is then substituted by the high resolution panchromatic image to render a new image in RGB, which is referred as the fused image. This is also called as a sharpened image. In the IHS transformation the three bands of the lower resolution image is utilized to translate it into the IHS space. Then, a contrast stretch is applied to the high resolution image, so that the stretched image has the same variance and average as the intensity component image. The stretched image that is the higher resolution image substitutes the intensity component before the image is metamorphosed to original color image.

As explained by Te-Ming et.al, saturation compensation can be accomplished by simply shifting so to $S^{\prime}$ or by multiplying with $\left(1+\Delta \mathrm{S} / \mathrm{S}_{0}\right)$. That is [5]:

$$
\left[\begin{array}{l}
\mathrm{R}_{\mathrm{s}} \\
\mathrm{G}_{\mathrm{s}} \\
\mathrm{B}_{\mathrm{s}}
\end{array}\right]=\left[\begin{array}{c}
\text { Pan } \\
\mathrm{Pan} \\
\operatorname{Pan}
\end{array}\right]+\left(1+\frac{\Delta \mathrm{S}}{\mathrm{S}_{0}}\right)\left[\begin{array}{cc}
-1 / \sqrt{2} & 1 / \sqrt{2} \\
-1 / \sqrt{2} & -1 / \sqrt{2} \\
\sqrt{2} & 0
\end{array}\right]\left[\begin{array}{l}
\nu 1_{0} \\
\nu 2_{0}
\end{array}\right],
$$

It is made computationally efficient by [7] :

$$
\begin{aligned}
{\left[\begin{array}{c}
\mathrm{R}_{\mathrm{s}} \\
\mathrm{G}_{\mathrm{s}} \\
\mathrm{B}_{\mathrm{s}}
\end{array}\right] } & =\left[\begin{array}{ccc}
1 & -1 / \sqrt{2} & 1 / \sqrt{2} \\
1 & -1 / \sqrt{2} & -1 / \sqrt{2} \\
1 & \sqrt{2} & 0
\end{array}\right]\left[\begin{array}{c}
\text { Pan } \\
\gamma^{\prime} \cdot \nu 1_{0} \\
\gamma^{\prime} \cdot \nu 2_{0}
\end{array}\right] \\
& =\left[\begin{array}{ccc}
1 & -1 / \sqrt{2} & 1 / \sqrt{2} \\
1 & -1 / \sqrt{2} & -1 / \sqrt{2} \\
1 & \sqrt{2} & 0
\end{array}\right]\left[\begin{array}{c}
\gamma^{\prime} \cdot \mathrm{I}+\left(\mathrm{Pan}-\gamma^{\prime} \cdot \mathrm{I}\right) \\
\gamma^{\prime} \cdot \nu 1_{0} \\
\gamma^{\prime} \cdot \nu 2_{0}
\end{array}\right] \\
& =\gamma^{\prime} \cdot\left[\begin{array}{l}
\mathrm{R} \\
\mathrm{G} \\
\mathrm{B}
\end{array}\right]+\left[\begin{array}{c}
\gamma^{\prime \prime} \\
\gamma^{\prime \prime} \\
\gamma^{\prime \prime}
\end{array}\right],
\end{aligned}
$$

Eqn (9)

Where

$$
\gamma^{\prime}=\mathrm{I} / \mathrm{Pan}=1+\Delta \mathrm{S} / \mathrm{S}_{0} \text { and } \gamma^{\prime \prime}=\mathrm{Pan}-\gamma^{\prime} \cdot \mathrm{I},
$$

\section{EXPERIMENTAL RESULTS}

The following data presents thumbnails of the output images and also compares them using the parameters like Mean, Median, Standard deviation, Correlation coefficient, Difference in variance, Entropy and Universal image quality index (Qfactor).The methods are implemented on images (Hyderabad).

\subsection{Image quality assessment:}

Many image quality assessment algorithms have been shown to behave consistently when applied to distorted images created from the same original image, using the same type of radiometric and spectral characteristics [3].

A quantitative approach should measure the ability of fusion process to transfer all perceptually important information of input images into the output image as accurately as possible. However, quantitative performance assessment is a difficult issue due to the variety of different application requirements and the lack of a clearly defined ground-truth. A wide range of quantitative fusion assessment techniques is based on the initial concepts of image quality metrics (such as Entropy, DIV, Qfactor and C.C) which are already used to compare quality of two different images in image processing applications.

\subsubsection{Qualitative analysis}

The most reliable judgment of image quality assessment is subjective rating by human observer which is known as qualitative analysis. Qualitative analysis involves visual comparison of color between original Multi Spectral and fused images, and the spatial detail between original Panchromatic and fused images. This method depends on the observers' experiences or bias thus some uncertainty is involved.

\subsubsection{Quantitative analysis}

Quantitative approaches involve a set of predefined quality indicators for measuring the spectral and spatial similarities between the fused image and the original Multi Spectral and/or Panchromatic .Amongst all developed objective quality metrics, Entropy, DIV, UQI and C.C are some of the most widely applied metrics.

\subsection{Entropy}

Entropy is a measure of information content of an image and is usually applied in image processing methods as a mean for measuring the information and complexity of images.

The Entropy of an image can be calculated by [8]: 


$$
\begin{aligned}
& \text { Entropy }=-\sum p_{i} \cdot \log _{2} p_{i} \\
& p_{i}=\operatorname{sum}(\text { image }==i) / N
\end{aligned}
$$

Where $p$ is the estimated probability density function (normalized pixel intensity histogram) of the selected image region.

For evaluating the quality of image fusion, the change in Entropy index is applied as quality metric. So we considered the change in Entropy index of each band of images before and after fusion as a metric for quality control:

$$
R_{E}=\text { Entropy }_{\text {Fuse dimage }}-\text { Entropy }_{\text {initia limage }}
$$

It is obvious that when no change occurs in information content of images or both input images (initial and fused image) are the same, the Entropy index RE is equal to 0 .

\subsection{Difference in Variance}

DIV inspects fusion quality over the whole image which means difference in variances relative to the original one [8]:

$$
D I V=\frac{\sigma_{M S}^{2}-\sigma_{F M S}^{2}}{\sigma_{M S}^{2}}
$$

Eqn (12)

Where $\sigma_{M S}^{2}$ is the variance of the original image and $\sigma_{F M S}^{2}$ is the variance of the fused image. This index presents the decrease or increase of information content during fusion process and would be positive for decreasing and negative for increasing change of information.

\subsection{Correlation Coefficient}

Correlation coefficient quantifies the closeness between two images. The correlation coefficient is computed using the following equation [8]:

$$
C . C=\frac{\sum_{l}^{N} \sum_{l}^{M}(x-\bar{x}) \cdot(y-\bar{y})}{\sqrt{\sum_{l}^{N} \sum_{l}^{M}(x-\bar{x})^{2}} \sum_{l}^{N} \sum_{l}^{M} \cdot(y-\bar{y})^{2}}
$$

Eqn (13)

The correlation coefficient value ranges from -1 to 1 , where the value +1 indicates that two images are highly correlated and are very close to each other. The value -1 indicates that the images are exactly opposite to each other.

\subsection{Universal Image Quality Index [Q- factor]}

Universal Image quality index is a new type of quality index proven to be better than widely used distortion metric mean squared error. Instead of using traditional error summation methods, this index is designed by modeling the image distortion as a combination of three factors: loss of correlation, luminance distortion, and contrast distortion [3].

Its definition is

$$
\text { Let } \mathbf{x}=\left\{x_{i} \mid i=1,2, \cdots, N\right\} \text { and } \mathbf{y}=\left\{y_{i} \mid i=1,2, \cdots, N\right\}
$$

be the original and the test image signals, respectively. quality index is defined as

$$
Q=\frac{4 \sigma_{x y} \bar{x} \bar{y}}{\left(\sigma_{x}^{2}+\sigma_{y}^{2}\right)\left[(\bar{x})^{2}+(\bar{y})^{2}\right]},
$$

where

$$
\bar{x}=\frac{1}{N} \sum_{i=1}^{N} x_{i}, \quad \bar{y}=\frac{1}{N} \sum_{i=1}^{N} y_{i},
$$

$$
\begin{gathered}
\sigma_{x}^{2}=\frac{1}{N-1} \sum_{i=1}^{N}\left(x_{i}-\bar{x}\right)^{2}, \quad \sigma_{y}^{2}=\frac{1}{N-1} \sum_{i=1}^{N}\left(y_{i}-\bar{y}\right)^{2} \\
\sigma_{x y}=\frac{1}{N-1} \sum_{i=1}^{N}\left(x_{i}-\bar{x}\right)\left(y_{i}-\bar{y}\right) .
\end{gathered}
$$

The dynamic range of $\mathrm{Q}$ is $[-1,1]$. The best value 1 is obtained only if $\mathrm{X}=\mathrm{Y}$ and the lowest value -1 occurs when $y_{i}=2 \bar{x}-x_{i}$

for all $\mathrm{i}=0,1,2 \ldots \mathrm{N}$.

\subsection{SSIM}

Structural Similarity is based on the idea that the human visual system is highly adapted to process structural information and the algorithm attempts to measure the change in this information between and reference and distorted image. Based on numerous tests, SSIM does a much better job at quantifying subjective image quality than MSE or PSNR [8].

At a high level, SSIM attempts to measure the change in luminance, contrast, and structure in an image. Luminance is modeled as average pixel intensity, contrast by the variance between the reference and distorted image, and structure by the cross-correlation between the 2 images. The resulting values are combined (using exponents referred to as alpha, beta, and gamma) and averaged to generate a final SSIM index value.

\subsection{For the satellite images}

Table 1: Basic qualitative analysis

\begin{tabular}{|l|l|l|l|}
\hline TECHNIQUES & \multicolumn{3}{|l|}{ PROPOSED METHOD } \\
\hline BASIC STATUS & B1 & B2 & B3 \\
\hline MIN & 0 & 0 & 0 \\
\hline MAX & 251 & 235 & 236 \\
\hline MEAN & 77.81 & 50.33 & 53.84 \\
\hline $\begin{array}{l}\text { STANDARD } \\
\text { DEVIATION }\end{array}$ & 28.48 & 34.17 & 30.96 \\
\hline
\end{tabular}


Table 2: Universal quality index:

\begin{tabular}{|l|l|l|l|}
\hline $\begin{array}{l}\text { PROPOSED } \\
\text { METHOD }\end{array}$ & $\begin{array}{l}\text { BAND1 = } \\
0.7917\end{array}$ & $\begin{array}{l}\text { BAND2 } \\
0.8914\end{array}$ & $\begin{array}{l}\text { BAND3 = } \\
0.8297\end{array}$ \\
\hline
\end{tabular}

Table 3: Difference in variance:

\begin{tabular}{|l|l|}
\hline PROPOSED METHOD & 4.1222 \\
\hline
\end{tabular}

Table 4: Entropy:

\begin{tabular}{|l|l|}
\hline PROPOSED METHOD & 0.0022 \\
\hline
\end{tabular}

Table 5: Correlation coefficient:

\begin{tabular}{|l|l|}
\hline PROPOSED METHOD & 0.9022 \\
\hline
\end{tabular}

Table 6: SSIM:

\begin{tabular}{|l|l|}
\hline PROPOSED METHOD & 0.712 \\
\hline
\end{tabular}

\subsection{For the normal images}

Table 7: Basic qualitative analysis:

\begin{tabular}{|l|l|l|l|}
\hline TECHNIQUES & \multicolumn{3}{|l|}{ PROPOSED METHOD } \\
\hline BASIC STATUS & B1 & B2 & B3 \\
\hline MIN & 2 & 19 & 0 \\
\hline MAX & 255 & 255 & 255 \\
\hline MEAN & 95.65 & 103.19 & 92.55 \\
\hline $\begin{array}{l}\text { STANDARD } \\
\text { DEVIATION }\end{array}$ & 71.30 & 65.66 & 64.40 \\
\hline
\end{tabular}

Table 8: Universal quality index:

\begin{tabular}{|l|l|l|l|}
\hline $\begin{array}{l}\text { PROPOSED } \\
\text { METHOD }\end{array}$ & $\begin{array}{l}\text { BAND1 } \\
0.9463\end{array}$ & $\begin{array}{l}\text { BAND 2 } \\
=0.7601\end{array}$ & $\begin{array}{l}\text { BAND3 } \\
0.8618\end{array}$ \\
\hline
\end{tabular}

Table 9: Difference in variance:

\begin{tabular}{|c|c|}
\hline PROPOSED METHOD & 3.6222 \\
\hline \multicolumn{2}{|c|}{ Table 10: Entropy } \\
\hline PROPOSED METHOD & 0.0002 \\
\hline \multicolumn{2}{|c|}{ Table 11: Correlation coefficient: } \\
\hline PROPOSED METHOD & 0.8902 \\
\hline \multicolumn{2}{|c|}{ Table 12: SSIM: } \\
\hline PROPOSED METHOD & 0.699 \\
\hline
\end{tabular}

\subsection{Fusion examples with satellite images:}

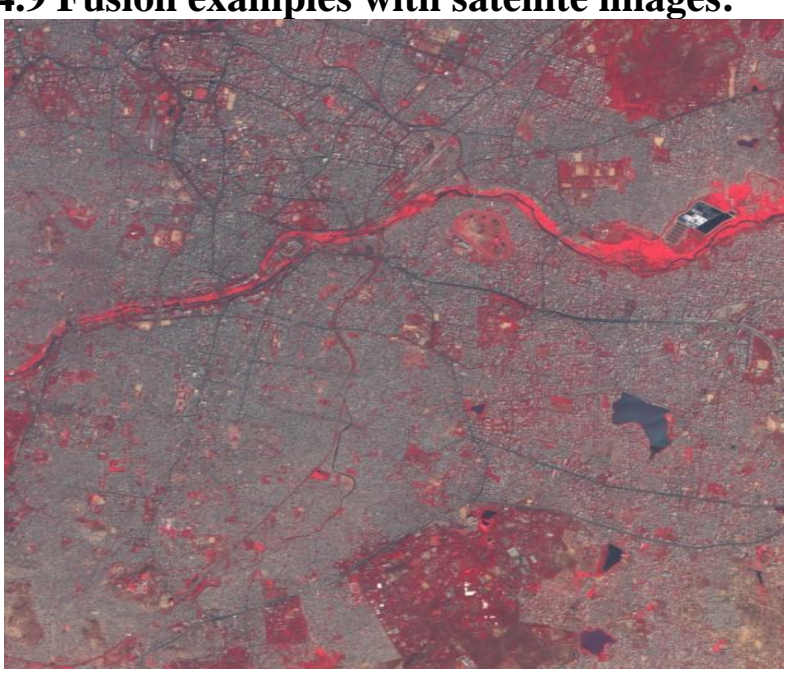

Fig 1: Multispectral Image

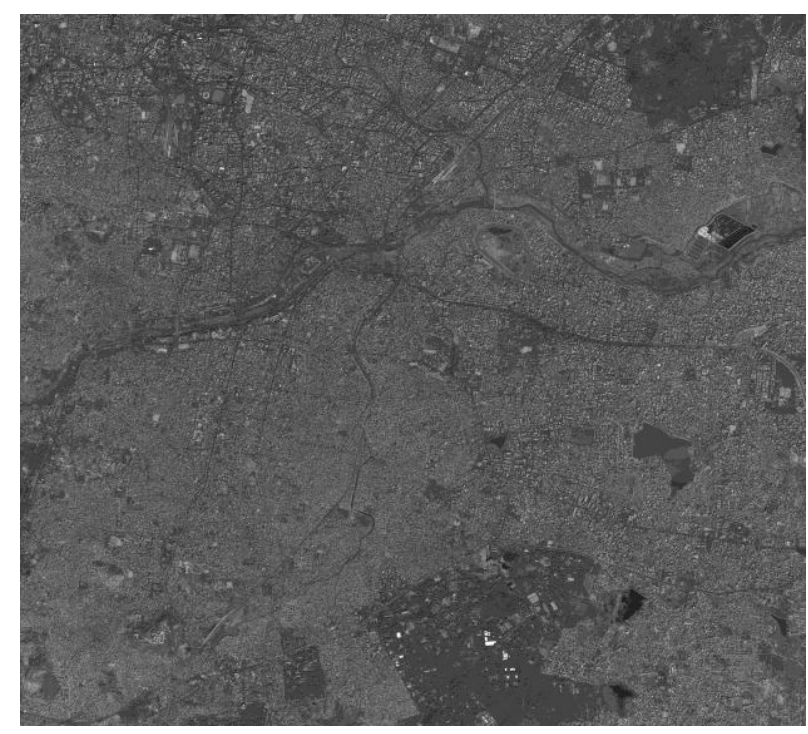

Fig 2: Panchromatic Image

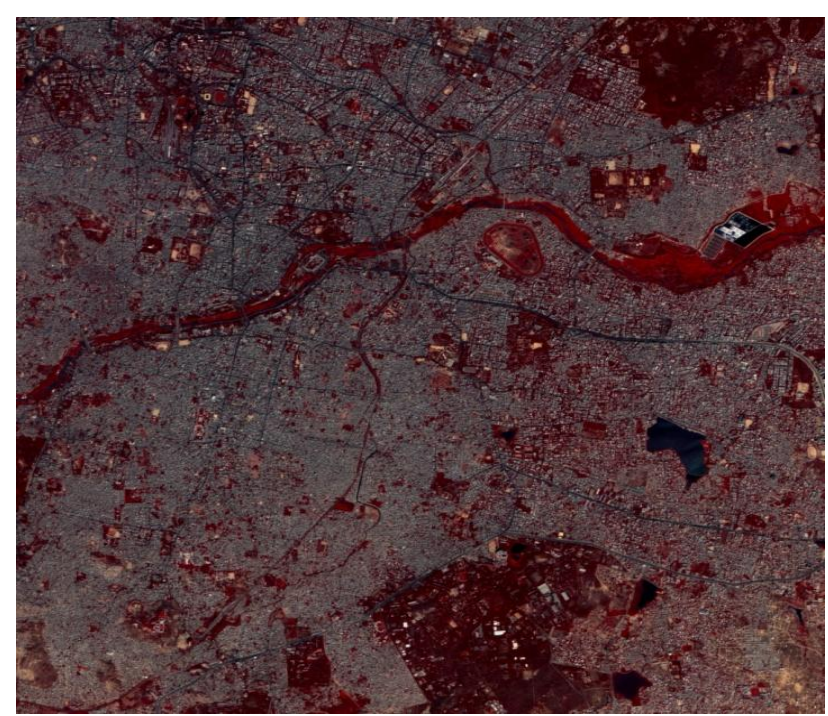

Fig 3: Proposed His Method 


\subsection{Fusion examples of normal images:}

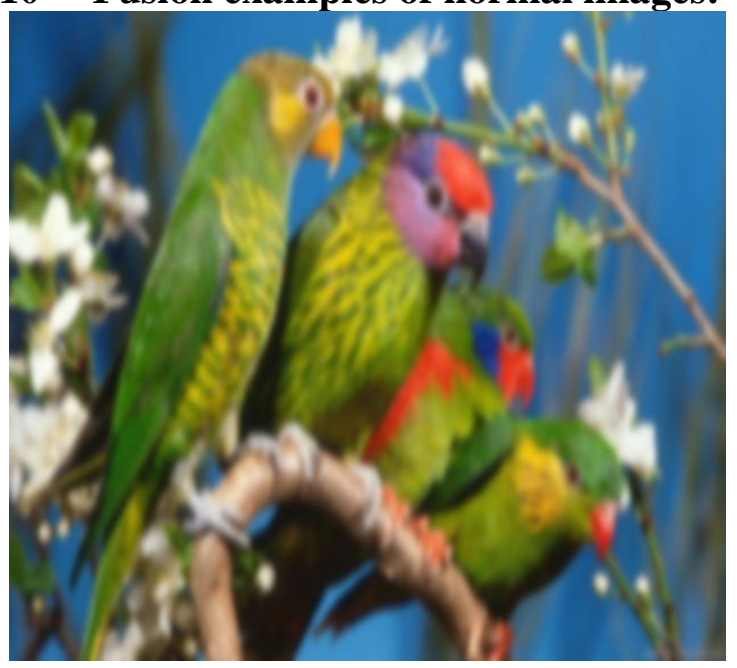

Fig 4: Multispectral Image

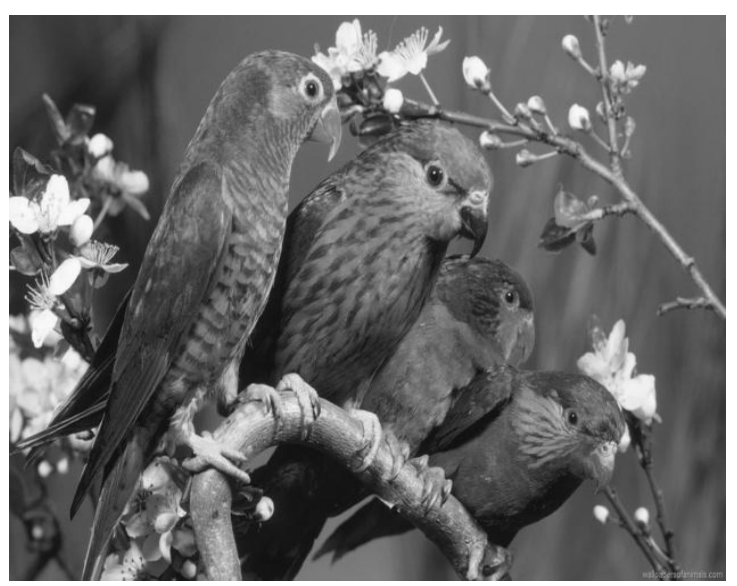

Fig 5: Panchromatic Image

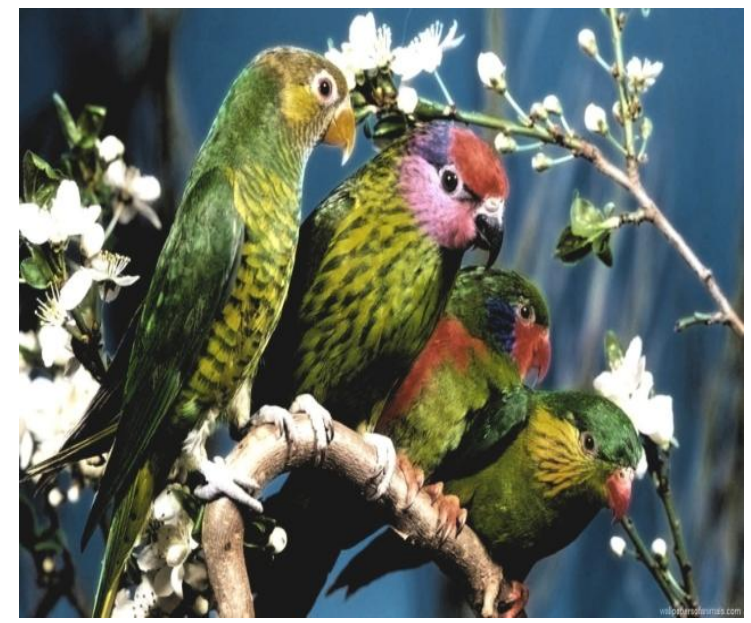

Fig 6: Proposed His Method
The new proposed method will produce the best result compare than the other existing methods with satellite images and as well as normal images.

\section{CONCLUSION}

It can be concluded from experimental results that the selection of a particular fusion methods depends on the application at hand. In case of fusing for urban areas spatial resolution is of importance and for agricultural fields spectral is more important than spatial. The proposed method is applicable for both normal images and satellite images. For the comparison finally conclude that the proposed method is the best one other than existing methods. The proposed method is related to the multiple linear regressions. Using various image quality assessment methods also conclude that the proposed method is better than the other existing HIS image fusion methods.

\section{REFERENCES}

[1] T. M. Tu, S. C. Su, H. C. Shyu, and P. S. Huang, "A new look at IHS-like image fusion methods," Inf. Fusion, vol. 2, no. 3, pp. 177-186, 2001.

[2] Wang Z., Ziou D., Armenakis C., Li D., and LiQ. "A Comparative Analysis of Image Fusion Methods". IEEE Transactions on Geoscience and Remote Sensing, Vol. 43, No. 6, June 2005 pp.1391-1402.

[3] Liao Y. C., Wang T. Y. and Zheng W. T., 1998. "Quality Analysis of Synthesized High Resolution Multispectral Imagery".

[4] Gonzalez, R.C. And Woods, R.E., 2001. "Digital Image Processing “. Prentice Hall.

[5] Firouz Abdullah Al-Wassai1, N.V. Kalyankar, Ali A. AlZuky, "The IHS Transformations Based Image Fusion," International Journal of Remote Sensing, Vol. 20, no. 3 , JULY 19, 2011.

[6] Wen Doua, Yunhao Chenb," An improved HIS image fusion method with high spectral fidelity," The International Archives of the Photogrammetric, Remote Sensing and Spatial Information Sciences. Vol. XXXVII. Part B7, no. 3, pp. 1253-1256, June 2008

[7] Ming Tuet.al. ,Efficient intensity-hue-saturation-based image fusion with saturation compensation ",IEEE Transactions on Optical engineering, Vol. 40, no. 5, pp. 720-728 ,May 2001.

[8] Sascha Klonus and Manfred Ehlers," Performance of evaluation methods in image fusion", 12th International Conference on Information Fusion, Vol. 16,pp. 14091416,July 6-9, 2009. 